\title{
A Clinico Microbiological Study of Diabetic Foot Ulcers
}

\author{
P. Meganathan ${ }^{1}$ and I.M. Rejitha ${ }^{2 *}$ \\ ${ }^{1}$ Kauvery Hospitals, Trichy, India \\ ${ }^{2}$ Tirunelveli Medical College, Tirunelveli, India \\ *Corresponding author
}

\section{A B S T R A C T}

\begin{tabular}{|l|}
\hline K e y w o r d s \\
Microbiological, \\
Cotrimoxazole, \\
Cefotaxime
\end{tabular}

Infection complicates the pathological picture of diabetic foot and plays a main role in development of moist gangrene. A total sample size of 100 patients with diabetic foot ulcer was included in this cross sectional study. A detailed history was obtained and assessment of clinical symptoms and signs were made. Discharge from the lesions, pus aspirates from the abscesses and debrided necrotic materials were collected. Aerobic culture anerobic culture and antibiotic susceptibility testing was done. Males were the predominant group in this study $66 \%$ as compared to females 34\%. Most of the patients (98\%) had type 2 Diabetes Mellitus. Around 50\% of the patients had Diabetes Mellitus for less than 5 years and the rest had Diabetes Mellitus for a period more than five years. Poor glycemic control was present in $90 \%$ cases and was a significant risk factor in relation to foot ulceration. Out of 100 samples 95 samples were culture positive. From the 95 culture positive patients 130 isolates were isolated as average 1.36 isolates per patient. The majority of the patients were infected with aerobes only. (89\%) Both aerobic and anaerobic organism could be isolated in the remaining patients. (11\%) Gram positive organisms were isolated in 53 patients. Staphylococcus aerues was the commonest isolate. Resistance to cotrimoxazole, cefotaxime, gentamycin was noticed among the gram negative bacteria.

\section{Introduction}

Diabetes mellitus is recognized as an epidemic in the Asian sub-continent affecting nearly 25 millions in India alone ${ }^{1}$. Foot disorders are a leading cause of hospitalization for patients with diabetes ${ }^{2}$. Ulceration of the foot is the precursor to approximately $85 \%$ of all diabetic amputations and it is estimated that $14.20 \%$ of patients with foot ulcers will have to undergo amputation.

The triad of problem leading on the diabetic foot is neuropathy, vascular changes and infections, which constitute the diabetic foot syndrome $^{3}$. Infection complicates the pathological picture of diabetic foot and plays a main role in development of moist gangrene. This study was done to detect the prevalence of diabetic foot, to know the risk factors for developing diabetic foot ulcers, microbial profile in diabetic wound, common susceptibility pattern to antibiotics and presence of multiple drug resistant organisms in diabetic foot.

\section{Materials and Methods}

A total sample size of 100 patients with diabetic foot ulcer (Both Outpatients and 
Inpatients) who were treated at Government Medical College Hospital Thoothukudi were included in this cross sectional study. A detailed history was obtained and assessment of clinical symptoms and signs were made.

Grading of foot lesions was as follows depending on the severity of lesions ${ }^{3}$

\section{Wagner ulcer classification systems}

Grade: 0: No open lesions; may have deformity or cellulites

Grade: 1: Superficial diabetic ulcer (Partial or full thickness)

Grade: 2: ulcer extension to ligament, tendon, joint capsule or deep facia without abscess or osteomyelitis

Grade: 3: Deep ulcer with abscess, osteomyelitis or joint sepsis

Grade: 4: Gangrene localized to portion to forefoot or heel

Grade: 5: Extensive gangrenous involvement

Osteomyelitis diagnosed on suggestive changes in the radiographs and bone scans. All cases were monitored until discharge from the hospital

Discharge from the lesions were collected with sterile swabs after the surface of the wound had been washed vigorously by saline, pus aspirates from the abscesses and debrided necrotic materials were collected.

\section{Microbiological Methods}

A gram stained direct smear of the specimen was examined. The specimen were cultured on blood agar and Mac Conkey agar for aerobic culture and incubated at $37 \mathrm{C}$ overnight. The bacterial isolates were identified by conventional biochemical tests.

\section{Susceptibility testing}

Anti-microbial susceptibility testing of isolates was performed by the standard disc diffusion method as recommended by the Clinical Laboratory Standards Institute

\section{Anaerobic culture}

Tissue bits, were inoculated into Robertson's Cooked Meat Media at the bed side, then the subculture was done on to blood agar plate, neomycin blood agar plate and kept in an anaerobic jar. Anaerobiasis maintained using gaspak (commercially purchased from Himedia). After incubation for 48 hours Bacteroides was confirmed by subculturing on to bacteroides bile esculin agar and observing the growth. Organism was identified by gram stain, microscopic and colony morphology and results of aerotolerance tests. The plates were incubated for another 48 hours to identify slow growing organisms. RCM was incubated at $37^{\circ} \mathrm{C}$ and observed for 7 days.

\section{Results and Discussion}

Pus samples were collected from 100 patients with diabetic foot ulcers Males were the predominant group in this study $66 \%$ as compared to females $34 \%$. This might be due to reduced immunity in old age and the increased duration of diabetes mellitus.

Most of the patients (98\%) had type 2 Diabetes Mellitus,

Around $50 \%$ of the patients had Diabetes Mellitus for less than 5 years and the rest had Diabetes Mellitus for a period more than five years. This could be because most of the patients were in the lower socio economic group and were illiterates having low level of 
awareness regarding diabetes. Many of them had their blood sugar checked at the time of admission for a non-healing ulcer.

The ulcers were either a non-healing ulcer followed by a trauma or began as a cellulitis initially, or were infections in a previous healed ulcer.

64\% patients had Neuropathy, 24\% had Nephropathy, 20\% had Peripheral Vascular Disease, Osteomyelitis was present in $22 \%$ of subjects.

Poor glycemic control was present in $90 \%$ cases and was a significant risk factor in relation to foot ulceration.

53\% patients had hypertension, this is similar to the study by Khalid et al., ${ }^{4}$ were 53 out of 116 patients included in the study had hypertension.

Awareness about risk factors regarding foot care was lacking and only $7 \%$ of patients were aware about the foot care.
The most common anatomical position of the ulcer was in the ball of the great toe $(30 \%)$ followed by the sole of the foot $14 \%$. It is mainly due to the high pressure over the ball of the great than other pressure points of the foot.

In the study conducted by Daivd G. Armstrong et al., ${ }^{5} 22 \%$ of diabetic foot ulcers were occurred in the ball of the great toe.

$76 \%$ of the patients in this study had grade 2 diabetic ulcers, In the study by Rooh Ulmugreem et al., ${ }^{6}$ the most of the patients had grade 3 ulcer while LIanes ${ }^{7}$ and Balderas ${ }^{8}$ have reported grade $2 / 3$ and grade 2 ulcer respectively in their study.

In this study 58 cases had previous history of ulcer which is comparable to other studies Khalid et al., ${ }^{4} 18$ cases had past history of amputation, $11 \%$ of the cases had necrotic ulcers, about $24 \%$ of case underwent surgical debridement and 22\% underwent amputation. All the cases received appropriate wound dressing and medical management (Table 1 and 2).

Table.1 Duration of the diabetes mellitus

\begin{tabular}{|c|c|}
\hline Duration (in years) & No of cases \\
\hline 0-1 Years & 14 \\
\hline $1-2$ Years & 12 \\
\hline $2-4$ Years & 18 \\
\hline $4-6$ Years & 14 \\
\hline 6-8 Years & 8 \\
\hline 8-10 Years & 20 \\
\hline $10-12$ Years & 4 \\
\hline$>12$ Years & 10 \\
\hline
\end{tabular}


Table.2 Profile of isolated organisms from infected foot ulcers in diabetic patient's specimens $(\mathrm{n}=130)$

\begin{tabular}{|l|c|c|}
\hline \multicolumn{1}{|c|}{ Organisms } & No of Isolates & Percentage \\
\hline Staphylococcus aureus & 41 & $\mathbf{3 1 . 2}$ \\
\hline Proteus Species & 26 & $\mathbf{2 1 . 0}$ \\
\hline E. coli & 20 & $\mathbf{1 5 . 2}$ \\
\hline Klebsiella species & 11 & $\mathbf{8 . 4}$ \\
\hline Citrobactor Species & 2 & $\mathbf{1 . 5}$ \\
\hline Pseudomonas aeruginosa & 2 & $\mathbf{1 . 5}$ \\
\hline Diptheriods & 4 & $\mathbf{3 . 0}$ \\
\hline CONS & 8 & $\mathbf{6 . 1}$ \\
\hline Bacteroids & 9 & $\mathbf{6 . 8}$ \\
\hline Peptostreptococci & 5 & $\mathbf{3 . 8}$ \\
\hline Candida & $\mathbf{2}$ & $\mathbf{1 . 5}$ \\
\hline
\end{tabular}

Out of 100 samples 95 samples were culture positive.

From the 95 culture positive patients 130 isolates were isolated as average 1.36 isolates per patient.

The majority of the patients were infected with aerobes only. (89\%) Both aerobic and anaerobic organism could be isolated in the remaining patients. (11\%) Fewer recovery of anaerobes because only few patients had gangrene.

In the study done by Unachukwu et al., ${ }^{9}$ Aerobes and anaerobes constituted $95.4 \%$ and $4.6 \%$ of the total bacterial isolates respectively.

Gram positive organisms were isolated in 53 patients. Staphylococcus aerues was the commonest isolate being recovered in $41 \%$. This is similar to the other studies in Ali S et al., ${ }^{10}$, Rooh - ul - Muqeem et al., ${ }^{6}$, other organisms isolated were Proteus sp (26), E.coli (20), Kelbsiella sp 11, Citrobactor and Pseudomonas 2 each, Bacteroidssfagilis was isolated in 9 cases and peptostreptococci in 5 cases.

Antibiotic sensitivity testing revealed that most $S$. aureus isolated were sensitive to amikacin and ciprofloxacin. They were resistant to cotrimoxazole, erythromycin and penicillin. MRSA was notices among 15 of them. Among the GNB E-Coli was uniformly sensitive to Amikacin, Klebsiella and Proteus - most of them sensitive to amikacin and ciprofloxacin. Resistance to cotrimoxazole, cefotaxime, gentamycin was notices among these bacteria, the prevalence of multidrug resistant organisms was around $76 \%$. Patients with MDRO infected ulcer had significantly higher blood glucose levels than those with non MDRO infected ulcers.

15 of the E. coli, 10 among Klebsiellae and 17 isolates among Proteus were detected to be ESBL producers.

In the study Gadepalli et al., ${ }^{11}$ ESBL Production and methicillin resistance was noted in 44.7 and $56 \%$ of bacterial isolates respectively.

In conclusion, this study presents a comprehensive clinical and microbiological 
survey of infected diabetic foot ulcer in hospitalized patients. Lack of awareness, poor glycemic control, long duration of diabetes and neuropathy were the main risk factors in the causation of diabetic foot ulcers. In this study gram positive aerobes were the predominant isolates.

The present study confirms that infection with Multiple Drug Resistant organisms is extremely common in hospitalized patients with diabetic foot ulcers.

\section{References}

1. Dushy ant singh Gour, Amitvarma, Pratimagupta Diabetic foot in Uttaranchal JK Science 9, No.1, January - March 2007 18-20.

2. Robert G. Frykberg, DPM, MPH, David G. Armstrong, DP M, John Giurini, DPM, Diabetic foot disorders, a clinical practice guideline second printing by the American College of Foot and Ankle surgeons, Published by Data Trace Publishing Company, S2-S60

3. Anandi C, Alaguraja D, Natarajan V, Ramanathan M, Subramaniam CS, Thulasiram M, Sumithra S. Bacteriology of diabetic foot lesions. Indian $\mathrm{J}$ Med Microbiol [serial online] 2004 [cited 2009 Jan 22]; 22: 175-8

4. Khalid Mahmood, S. TehseenAkhtar, Abu Talib, BadarAbbasi, Siraj -ulsalekeen and lftikarhaider Naqvi. Clinical Profile and Management Outcome of Diabetic Foot Ulcer in a Tertiary Care Hospital Journal of the
College of Physicians and Surgeons Pakistan 2008, Vol. 18(7): 408-412

5. David G. Armstrong, DPM, Lawrence A. Lavery, DPM, MPH, Lawrence B. Harkless, DPM, Validation of a diabetic wound classification system. Diabetes Care, Vol 21 (5) May 1998: 855-859

6. Rooh - ul-Muqeem, Ahmed M, S. Evalution and management of diabetic foot according to Wagner's classification: a study of 100 cases. J Ayub Med Coll 2003; 15: 39-42

7. Lianes LR, Peng AC, Cauton-Valera R. Clinical, microbiological profile and outcome of diabetic patients with foot ulcers admitted ate the Quirino Memorial Medical Center 2000-May 2001. Pbilipp J Microbial Infect Dis 2001; 30: 101-7

8. Balderas JA, Oribio RU, racho V, LimAbrahan MA. Diabetic Extremity management by multidisciplinary care team: The Philippine General Hospital experience. Philipp J Intern Med 1999; 37: 246-52.

9. Unachukwu CN, Obunge OK, Odia OJ. The bacteriology of diabetic foot ulcers in Port Harcourt, Nigeria. Niger J Med. 2005 Apr-Jun; 14(2): 173-6

10. Ali S, Basit A, Sheikh T, Mumtaz S, Hydrie M, Diabetic foot ulcer - a prospective study. J Pak Med Assoc 2001; 51: 78-81.

11. RaviseharGadepalli R, Dhawan B, Sreenivas V, Kapil A, Ammini AC, Chaudhry B. A clinico - microbiological study of diabetic foot ulcers in an Indian Tertiary Care Hospital. Diabetes Care. 2006 Aug; 29(8): 1727-32

\section{How to cite this article:}

Meganathan, P., and Rejitha, I.M. 2019. A Clinico Microbiological Study of Diabetic Foot Ulcers. Int.J.Curr.Microbiol.App.Sci. 8(08): 439-443. doi: https://doi.org/10.20546/ijcmas.2019.808.049 